\title{
Modifizierte Herpesviren im Kampf gegen das Melanom
}

\section{Erstmals wurden in einer klinischen Phase-III-Studie die Wirksamkeit und Verträglichkeit einer onkolytischen Immuntherapie bei Patienten mit einem nicht resezierbaren Melanom untersucht.}

\begin{abstract}
7 ielgerichtete Therapien auf molekularer Ebene und immuntherapeutische Ansätze leiteten eine neue Ära in der Behandlung von Melanomen ein. Neueste Strategien zielen auf die Induktion der Apoptose von Tumorzellen und/ oder die Auslösung einer protektiven, systemischen antitumoralen Immunantwort ab. Talimogene Laherparepvec (T-VEC) ist ein erster Vertreter onkolytischer Viren auf der Basis genetisch modifizierter Herpes-simplex-Viren Typ 1 (HSV-1), die sich selektiv in Tumorzellen replizieren, den Untergang der malignen Zellen einleiten und eine systemische antitumorale Immunantwort auslösen.
\end{abstract}

T-VEC demonstrierte in früheren Studien antitumorale Aktivität und Expression von GM-CSF. In einer einarmigen Phase-II-Studie erreichten unter dieser Therapieform Patienten mit einem $\mathrm{Me}$ lanom der Stadien III-IV eine Gesamtansprechrate von $26 \%$. Jetzt liegen die ersten Ergebnisse der Phase-III-Studie OPTiM vor, in der das dauerhafte Ansprechen (DRR) von Patienten mit einem nicht resezierbaren höhergradigen Melanom auf eine intraläsionale Injektion von T-VEC im Vergleich zu GM-CSF subkutan untersucht wurde.

Patienten unter T-VEC profitierten von einem signifikant günstigerem
DRR als die Vergleichsgruppe: 16,3\% gegenüber $2,1 \%$. Dieser Vorteil zeigte sich auch im medianen Gesamtüberleben: 23,3 Monate gegenüber 18,9 Monaten. Unter den unerwünschten Ereignissen dominierten in der T-VECGruppe Fatigue, Schüttelfrost und Pyrexie. Cellulitis als höhergradiges unerwünschtes Ereignis trat bei 2,1\% der Patienten auf.

Fazit: Mit T-VEC steht die erste onkolytische Immuntherapie zur Verfügung, die im Rahmen einer klinischen PhaseIII-Studie einen therapeutischen Nutzen beim Melanom demonstrierte. Der positive therapeutische Effekt war besonders ausgeprägt bei unbehandelten Patienten in den Krankheitsstadien IIIB, IIIC oder IVM1a.

Wolfgang Zimmermann

Andtbacka RHI et al. Talimogene Laherparepvec improves durable response rate in patients with advanced melanoma. J Clin Oncol. 2015; 33: 2780-8

\section{Hier steht eine Anzeige.}

\section{量 Springer}

